\title{
Protein kinase CK2 phosphorylates and upregulates Akt/PKB
}

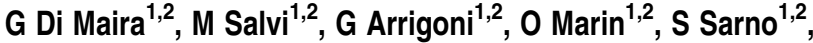 \\ F Brustolon $^{1,2}$, LA Pinna ${ }^{1,2}$ and M Ruzzene ${ }^{\star, 1,2}$ \\ 1 Venetian Institute of Molecular Medicine (VIMM), University of Padova, \\ Padova, Italy; \\ 2 Department of Biological Chemistry, University of Padova, Padova, Italy \\ * Corresponding author: M Ruzzene, Department of Biological Chemistry, \\ University of Padova, V.le G. Colombo, 3 - 35121 Padova, Italy. \\ Tel: + 39-049-8276112; Fax: + 39-049-8073310; \\ E-mail: maria.ruzzene@unipd.it
}

Received 22.7.04; revised 21.12.04; accepted 28.1.05; published online 01.4.05 Edited by JA Trapani

\begin{abstract}
Treatment of Jurkat cells with specific inhibitors of protein kinase CK2 induces apoptosis. Here we provide evidence that the antiapoptotic effect of CK2 can be at least partially mediated by upregulation of the Akt/PKB pathway. Such a conclusion is based on the following observations: (1) inhibition of CK2 by cell treatment with two structurally unrelated CK2 inhibitors induces downregulation of Akt/PKB, as judged from decreased phosphorylation of its physiological targets, and immunoprecipitate kinase assay; (2) similar results are observed upon reduction of CK2 catalytic subunit by the RNA-interference technique; (3) Akt/PKB Ser129 is phosphorylated by CK2 in vitro and in vivo; (4) such a phosphorylation of activated Akt/PKB correlates with a further increase in catalytic activity. These data disclose an unanticipated mechanism by which constitutive phosphorylation by CK2 may be required for maximal activation of Akt/PKB. Cell Death and Differentiation (2005) 12, 668-677.

doi:10.1038/sj.cdd. 4401604

Published online 1 April 2005
\end{abstract}

Keywords: CK2; casein kinase 2; CKII; inhibitors; Akt; protein kinase B

Abbreviations: CK2, casein kinase 2; GSK3, glycogen synthase kinase 3; IQA, 5-oxo-5,6-dihydroindolo-(1,2-a)quinazolin-7-yl]acetic acid; MALDI, matrix-assisted laser desorbtion and ionization; MS/MS, tandem mass spectrometry; PDK1, 3phosphoinositide-dependent protein kinase-1; $\mathrm{PH}$, pleckstrin homology; PI3-kinase, phosphatidylinositol 3-kinase; PKB, protein kinase B (Akt); PTEN, phosphatase and tensin homolog deleted on chromosome ten; RNAi, RNA interference; SGK, serum- and glucocorticoid-induced kinase; siRNA, short interfering RNA; TBB, 4,5,6,7-tetrabromobenzotriazole; WB, Western blot; w.t., wild type

\section{Introduction}

CK2, an acronym derived from the misnomer 'casein kinase 2', denotes a ubiquitous, essential and constitutively active protein kinase affecting phosphoacceptor sites that are specified by numerous acidic residues (either Glu/Asp or phosphorylated aminoacids), among which the one at position $\mathrm{n}+3$ plays the most crucial role. ${ }^{1}$

The high basal activity of the catalytic subunits ( $\alpha$ and/or $\alpha^{\prime}$ ) of CK2, either alone or in combination with a dimeric regulatory $\beta$-subunit to give an heterotetrameric holoenzyme, may reflect the striking pleiotropy of this kinase, whose present repertoire of substrates includes $>300$ proteins. ${ }^{1}$ These are implicated in a wide variety of cellular functions, including global processes as well as signaling pathways, a circumstance that could account for the requirement of active CK2 both in quiescent and in stimulated cells.

CK2 is invariably elevated in tumors as compared to normal tissues (reviewed in Guerra and Issinger ${ }^{2}$ and Tawfic et al. ${ }^{3}$ ) and unscheduled expression of its catalytic subunits is causative of transformation, especially under conditions that also alter the expression of oncogenes and tumor suppressor genes. ${ }^{4-6}$ Evidence has recently been accumulating that CK2 plays a prominent role in ensuring cell survival ${ }^{7,8}$ (and reviewed in Litchfield ${ }^{9}$ ) by counteracting apoptosis, ${ }^{10,11}$ a behavior which may account, at least in part, for its oncogenic potential. The mechanism(s), however, by which CK2 exerts its antiapoptotic role remain largely unexplored.

Evidence for the antiapoptotic role of CK2 has been obtained in LNCaP prostate cancer cells ${ }^{10}$ and in leukemia Jurkat $\mathrm{T}$ cells, ${ }^{11}$ both lacking the phosphatase and tensin homolog deleted on chromosome ten (PTEN) $\operatorname{protein}^{12,13}$ which removes phosphate from the D3 position of inositol phospholipids, and which is also one of the numerous substrates of CK2. ${ }^{14}$ Lack of PTEN maintains the phosphatidylinositol 3-kinase (PI3-kinase) pathway in a constitutively active state. Therefore, in both $\mathrm{LNCaP}$ and Jurkat cell lines, as in many leukemias and lymphomas where PTEN is also absent, protein kinase Akt (also known as PKB), whose activation is promoted by the $3^{\prime}$-phosphoinositide-dependent kinase-1 (PDK1), is constitutively upregulated (for reviews on Akt/PKB see Hanada et al., ${ }^{15}$ Scheid and Woodgett, ${ }^{16}$ and Nicholson and Anderson ${ }^{17}$ ). Akt exerts important antiapoptotic features, a property which is supposed to contribute to the tumor phenotype of PTEN-null cell lines. ${ }^{18}$

Interestingly, apoptosis of Jurkat cells can be promoted by two structurally unrelated specific inhibitors of CK2, which are inactive on either Akt or PDK1. ${ }^{19,20}$ This means that either CK2 operates through a pathway which is independent of Akt, or it can induce a hyperactivation of Akt beyond the level of constitutive activity displayed by this kinase in Jurkat and other PTEN-null cells.

To discriminate between these possibilities and to gain information about the apoptotic signaling pathways that might be affected by CK2, we have investigated the consequences of attenuating CK2 activity on the activity of Akt.

Here we provide evidence that in vitro and in vivo Akt is phosphorylated by CK2 at Ser129, with a concomitant increase in catalytic activity, thus disclosing the possibility 
that constitutive phosphorylation by CK2 is required for full activation of Akt.

\section{Results}

\section{CK2 inhibitors induce downregulation of Akt}

In order to gain information about the mechanism by which CK2 exerts its antiapoptotic potential, we checked whether treatment of Jurkat cells with two selective inhibitors of CK2, 4,5,6,7-tetrabromobenzotriazole (TBB) and 5-oxo-5,6-dihydroindolo-(1,2-a)quinazolin-7-yl]acetic acid (IQA), had any effect on endogenous Akt activity. For this purpose, the phosphorylation level of Akt-specific targets was determined using phospho-specific antibodies. As a positive control for Akt inhibition, we treated cells with LY294002, a well-known inhibitor of PI3-kinase. Under our conditions, while LY294002 was totally ineffective on CK2 endogenous activity, both TBB and IQA markedly inhibited CK2 ( ${ }^{11,20}$ and Figure 1a), and, as expected, ${ }^{11,20}$ induced cell death by apoptosis, as monitored by MTT viability assay (not shown) and by the cleavage of the caspase substrate PARP (Figure 1b). As far as the Akt signaling pathway is concerned, treatment of Jurkat cells with both CK2 inhibitors resulted in a marked drop in the phosphorylation level of GSK3 $\beta$ Ser9, FKHR Ser256, and AFX Ser193 (Figure 1c). Note that phosphorylation of PDK1, the upstream kinase responsible for canonical Akt regulation, is not altered by either TBB or IQA. Consequently, also phosphorylation of Akt Thr308, which is specifically targeted by PDK1, is only marginally reduced. A weak inhibition of Ser473 phosphorylation is also observed; this is negligible as compared to that promoted by the PI3-kinase inhibitor LY294002, and therefore not sufficient to justify the reduction of Akt activity monitored by the drop in substrate phosphorylation. Figure $1 \mathrm{c}$ also shows that the total amount of Akt and glycogen synthase kinase 3 (GSK3) proteins was not affected by the treatments.

We also measured directly the activity of Akt towards a synthetic peptide in an immunoprecipitate kinase assay, after cell incubation with the CK2 inhibitors. As shown in Figure 2, the catalytic activity of Akt was significantly reduced by treatments with both inhibitors, IQA and TBB, under conditions where comparable amounts of enzyme were present in the immunoprecipitates.

\section{Decreased levels of CK2 catalytic subunit correlate with inactivation of Akt}

In order to make sure that the detrimental effect of CK2 inhibitors on the activity of Akt was really mediated by blockage of CK2, rather than of other kinases, advantage has been taken of the RNA interference (RNAi) technique to achieve a nonpharmacological and strictly selective decrease in endogenous CK2 catalytic activity. LNCaP cells were used for these experiments (given the availability of simpler protocols to trigger RNA interference in adherent than in suspension cells). Similar to Jurkat, LNCaP cells are PTENnull and therefore Akt is constitutively activated. As shown in Figure 3a, the approach proved successful for diminishing the level of expression of CK2 $\alpha$ catalytic subunit to about $39 \%$. a

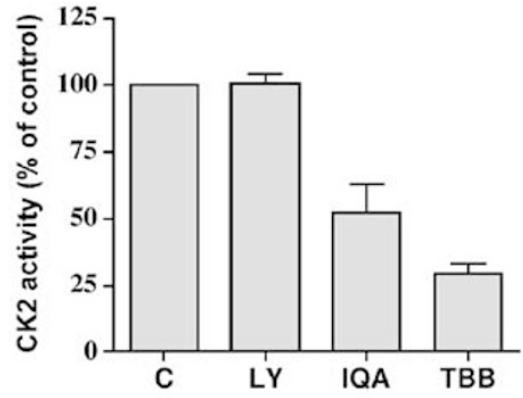

b

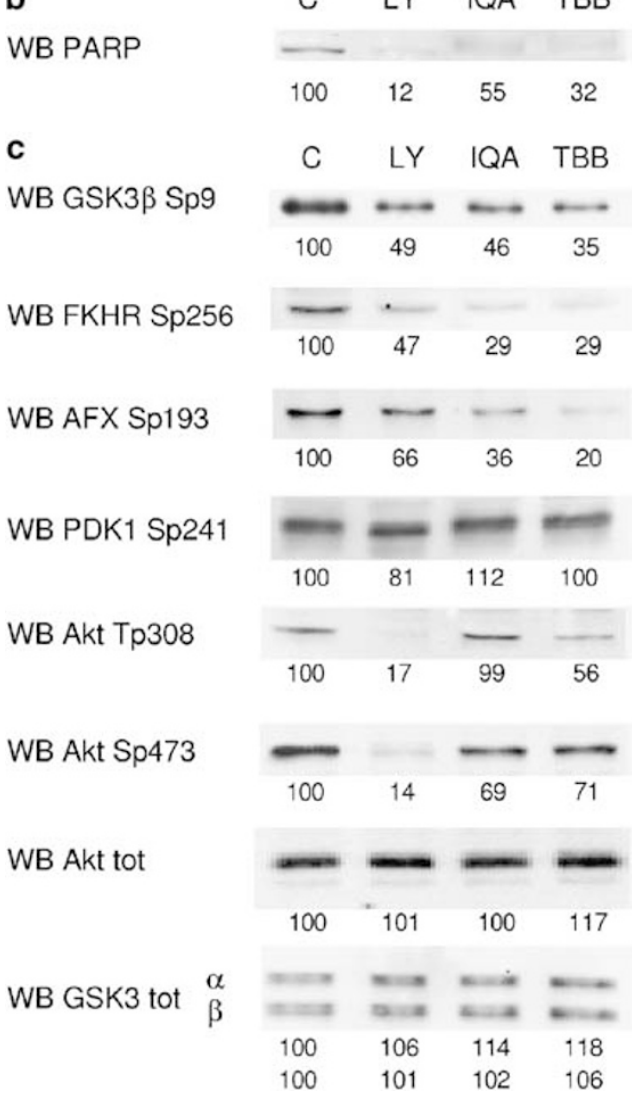

Figure 1 Downregulation of Akt pathway by CK2 inhibitors in Jurkat cells. Jurkat cells were treated for $4 \mathrm{~h}$ with CK2 inhibitors, as indicated. IQA was $40 \mu \mathrm{M}$, TBB was $25 \mu \mathrm{M}$. Where shown (LY), the PI3-kinase inhibitor LY294002 $(25 \mu \mathrm{M})$ was used as a control for Akt downregulation. (a) Activity of CK2 in cell lysates was measured towards the CK2-specific peptide substrate (bar graphs, mean values ( \pm S.D.) of three determinations). In all, $100 \%$ CK2 activity was assigned to lysate from control cells (C, $0.15 \mathrm{pmol} / \mathrm{min} / \mu \mathrm{g}$ protein). (c and b) $5 \mu \mathrm{g}$ (in the case of anti-Akt Sp473) or $20 \mu \mathrm{g}$ (for all the other antibodies) of total lysate proteins were analyzed by Western blot (WB) with the indicated antibodies. Band quantitation was obtained on a Kodak Image Station 440CF by analysis with the Kodak 1D Image software, and the values are reported below each band, assigning the value of 100 to the control cells (C)

This reduction results, as expected, in a substantial drop in CK2 catalytic activity (of about $36 \%$ ). As shown in Figure 3b, the knock down of the CK2 catalytic subunit is accompanied by a substantial reduction of Akt activity, as judged by immunoprecipitate kinase assay, or by the dephosphorylation degree of the endogenous targets GSK3 $\beta$ Ser9 and FKRH Ser256. Under these conditions, the GSK3 and Akt protein amounts were not affected, nor the Akt phosphorylation state 


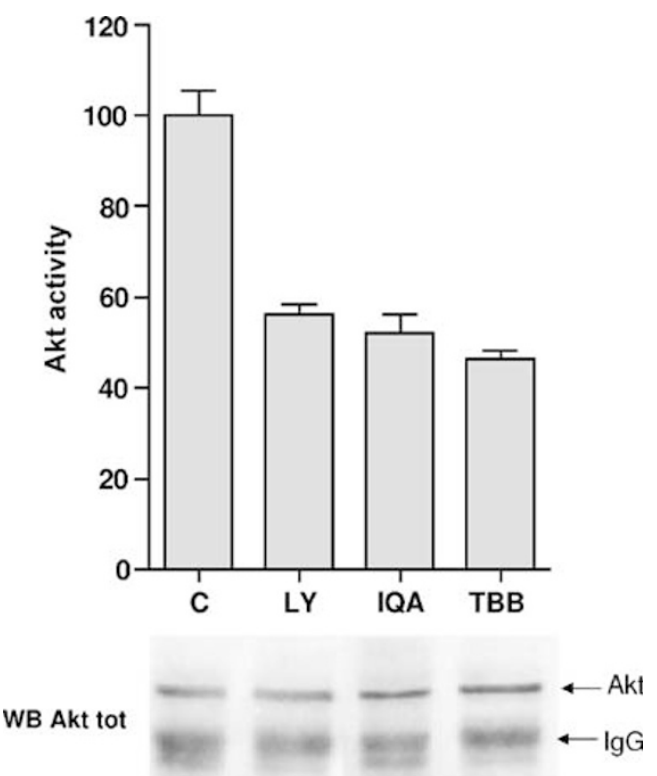

Figure 2 Akt immunoprecipitate kinase assay from Jurkat cells treated with CK2 inhibitors. Jurkat cells were treated for $4 \mathrm{~h}$ with CK2 inhibitors, as indicated. IQA was $40 \mu \mathrm{M}$, TBB was $25 \mu \mathrm{M}$. Where shown (LY), the PI3-kinase inhibitor LY294002 $(25 \mu \mathrm{M})$ was used as a control for Akt downregulation. Akt was immunoprecipitated from the cell lysate, and its activity was measured towards a peptide substrate, by incubation for $30 \mathrm{~min}$ in the presence of a radioactive phosphorylation mixture, as described in Materials and Methods; the radioactivity incorporated is reported as arbitrary units, assigning an activity of 100 to Akt immunoprecipitated from control cells $(C)$. Mean values $( \pm$ S.D.) from two separate experiments are shown. The amount of total Akt in each immunoprecipitate was monitored by Western blot, as shown in the bottom part of the figure

was significantly altered (not shown). This indicates that Akt activity is effectively downregulated in cells treated with short interfering RNA (siRNA) against CK2 catalytic subunit.

\section{CK2 phosphorylates and activates Akt in vitro}

The residues whose phosphorylation is known to be most responsible for Akt activation are Thr308 (in the activation loop) and Ser473 (in the hydrophobic domain outside the catalytic domain). The former is phosphorylated by PDK $1,{ }^{21}$ while Ser473 phosphorylation is still a matter of debate, one possibility being that this may occur autocatalytically. In any event, neither Ser473 nor Thr308 display the consensus required for CK2 phosphorylation, not to say that in PTEN-null cells both these residues are expected to be constitutively phosphorylated, irrespective of CK2. Akt includes, on the other hand, a few residues that display the consensus sequence for CK2-mediated phosphorylation, and we wanted to see if the in vivo activation of Akt by CK2 activity might be due, at least in part, to direct phosphorylation of Akt by CK2. To check this possibility, a number of in vitro experiments were performed where variably phosphorylated forms of Akt were subjected to phosphorylation by CK2. The results are shown in Figure 4. It can be seen that both an active form of Akt, previously phosphorylated at both Ser473 and Thr308, and an inactive unphosphorylated form are readily phosphorylated by the CK2 holoenzyme, and, even more, by CK2 $\alpha$ (compare lanes 2 and 5 with lanes 3 and 6 , respectively). a
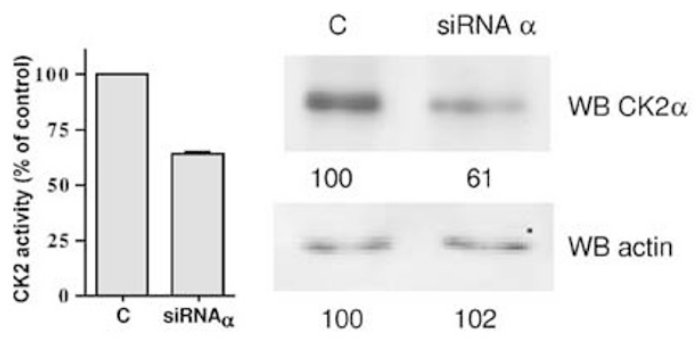

b
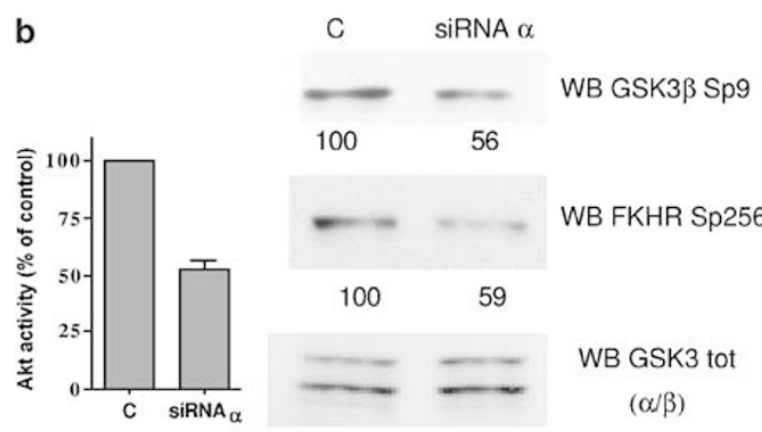

WB FKHR Sp256

100

59

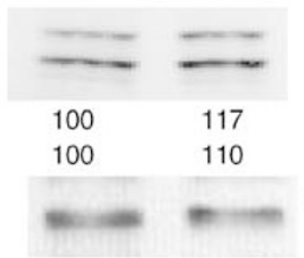

WB GSK3 tot

$(\alpha / \beta)$

WB Akt tot

100

98

Figure 3 Knock down of CK2 by the RNA interference technique and effect on Akt signaling. LNCaP cells were treated for $72 \mathrm{~h}$ with siRNA sequences targeting CK2 $\alpha$. When Western blot experiments are shown, band quantitation is reported below each band, assigning the value of 100 to control cells (C), treated with non silencing siRNA. (a) The bar graph shows the activity of CK2 in cell lysates, measured towards the CK2-specific peptide substrate (mean values $( \pm$ S.D.) of two determinations). $100 \%$ CK2 activity was assigned to lysate from control cells. The right part of the figure shows the Western blot ( $10 \mu \mathrm{g}$ of total proteins) with anti-CK2 $\alpha$ specific antibodies or antiactin, for normalization. (b) The bar graph shows the activity of Akt, measured towards a peptide substrate, after immunoprecipitation from cell lysates (mean values $( \pm$ S.D.) of two determinations). In all, $100 \%$ Akt activity was assigned to the Akt immunoprecipitated from control cells. The right part of the figure shows the Western blot $(10 \mu \mathrm{g}$ of total proteins) with the indicated antibodies

Interestingly, three related kinases with remarkable similarity to Akt, but lacking consensus sequences for CK2, serum- and glucocorticoid-induced kinase (SGK)-1, 2 and 3, are not phosphorylated by CK2 to any appreciable extent (not shown).

We wondered whether phosphorylation by CK2 might have any effect on Akt activity. We therefore performed experiments in which the activity of Akt, incubated or not with CK2 and Mg/ATP, was determined using either a peptide (Figure $5 \mathrm{a}$ ) or a protein (Figure $5 \mathrm{~b}$ ) substrate, containing the consensus for Akt (R/K-x-R/K-x-x-S/T) but not for CK2. The results demonstrate that CK2 promotes a $\sim 2$-fold hyperactivation of active Akt and that this effect is mediated by phosphorylation, as it is suppressed by the CK2-specific inhibitor TBB.

In the absence of previous phosphorylation at Thr308 and Ser473, Akt activity was undetectable with the peptide/protein substrates used, either before or after phosphorylation by CK2 (not shown); however, a weak signal was detected using the anti-phospho-Ser473 antibodies, and this was significantly 


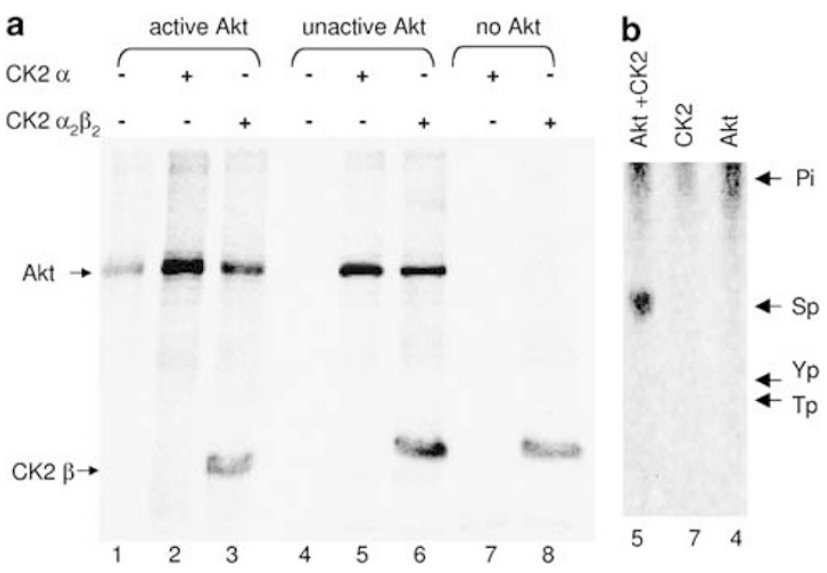

Figure 4 In vitro phosphorylation of Akt by CK2. Incubations were performed for $20 \mathrm{~min}$ in the presence of radioactive phosphorylation mixture (see Materials and Methods), with the indicated protein kinases. In all, $0.2 \mu \mathrm{g}$ of active or unactive Akt1 was used. Under the conditions used, the CK2 $\alpha(0.1 \mu \mathrm{g})$ or CK2 $\alpha_{2} \beta_{2}(0.02 \mu \mathrm{g})$ amounts were equally active towards casein substrate (not shown). (a) An autoradiography is shown of phospho-proteins resolved on SDS/ PAGE. Autophosphorylation of CK2 $\beta$ subunit is also visible. Stoichiometry of Akt1 phosphorylation was about $0.2 \mathrm{~mol}$ phosphate/mol protein. (b) Autoradiography of the high-voltage paper electrophoresis for the identification of the phospho-amino acid. Samples from lanes 4, 5, and 7 of panel a were hydrolyzed in $6 \mathrm{~N} \mathrm{HCl}$, and subjected to high-voltage paper electrophoresis, as described in Material and Methods, for the identification of the phospho-amino acid. An autoradiography of the paper is shown. The position of orthophosphate (Pi) and phospho-amino acids (Sp, Yp, and Tp), determined by cold standard migration, is indicated by the arrows

increased by CK2 (Figure 5c). Since the Ser473 site is not affected by CK2 (see below), this finding must reflect a CK2induced stimulation of Akt autophosphorylation activity.

\section{Ser129 is the major target for CK2 in Akt}

Akt1 contains six residues that fulfill the consensus sequence for CK2 (S/T-x-x-E/D), namely Thr92, Ser129, Thr146, Thr165, Ser259, and Ser431. All of these, with the exception of Ser129, are 'poor' phosphoacceptor sites, for having no additional acidic residues in the proximity of the target amino acids, a part from the one at position $n+3 .^{1}$ Conversely, Ser129 can be classified as a good CK2 target for having acidic residues at positions $n+3,+4,+6$, and -2 . Moreover, once phosphorylated it could prime the phosphorylation of Ser126.

Phosphorylation of threonyl residues was ruled out by phosphoaminoacid analysis showing that only phosphoserine could be isolated from Akt1 phosphoradiolabeled by CK2 (Figure 4b).

In order to identify the seryl residue(s) phosphorylated by CK2, recombinant Akt1 previously phosphorylated in vitro by CK2 was subjected to SDS/PAGE and its band was digested with trypsin in gel and analyzed by matrix-assisted laser desorbtion and ionizsation (MALDI) mass spectrometry. As shown in Figure 6a, a peptide with a molecular weight compatible with the triptic fragment [122-142] (2119.83 Da) was detected together with its possible monophosphorylated form $(2199.83 \mathrm{Da})$. To prove that the peptide with the higher molecular mass was actually due to a phosphorylation of the

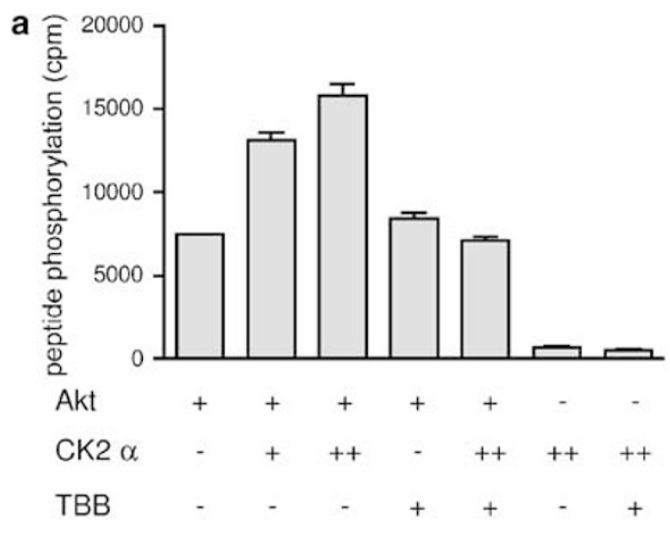

b

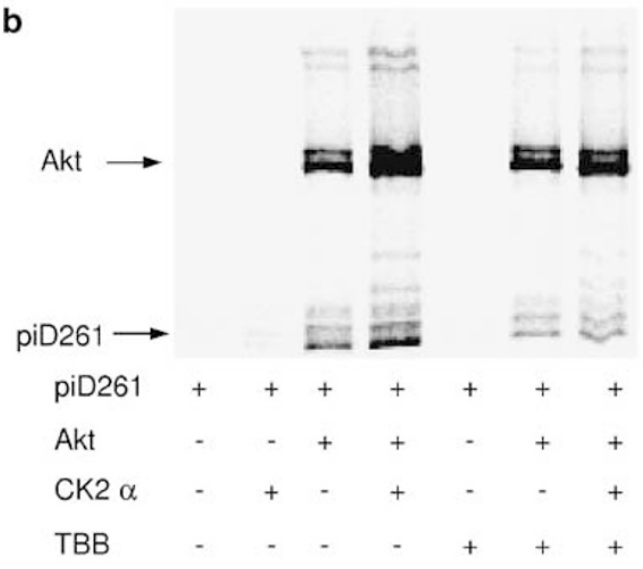

C

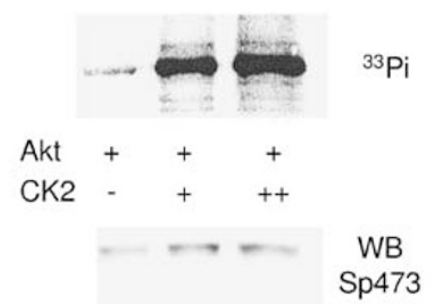

Figure 5 Effect of CK2 on the activity of Akt in vitro. (a) Active Akt $(0.2 \mu \mathrm{g})$ was incubated for $20 \mathrm{~min}$ with radioactive phosphorylation mixture in the absence (-) or in the presence of $0.05 \mu \mathrm{g}(+)$ or $0.1 \mu \mathrm{g}(++)$ CK2 $\alpha$. Where indicated, TBB was present at $1 \mu \mathrm{M}$ concentration. Akt activity measured towards a peptide substrate is shown as cpm incorporated (mean values $( \pm$ S.D.) from three separate experiments) (b) Active Akt $(0.2 \mu \mathrm{g})$ was incubated as described for panel a, but its activity was evaluated towards the protein substrate piD261 $(0.1 \mu \mathrm{g})$; an autoradiography is shown of phospho-proteins resolved on SDS/ PAGE. (c) Unactive Akt $(0.2 \mu \mathrm{g})$ was incubated for 20 min with radioactive phosphorylation mixture in the absence $(-)$ or in the presence of $0.05 \mu \mathrm{g}(+)$ or $0.1 \mu \mathrm{g}(++)$ CK2 $\alpha$; the incorporated radioactivity is shown in the upper part, while the degree of Ser473 phosphorylation detected by Western blot with the phospho-specific antibody is shown in the bottom part

peptide [122-142], the sample was chemically dephosphorylated and analyzed again by MALDI mass spectrometry. As shown in Figure $6 \mathrm{~b}$, the peak underwent the $98 \mathrm{Da}$ shift expected for $\beta$-elimination of phosphate from seryl/threonyl residue. The [122-142] sequence includes the best potential CK2 site (Ser129) together with other four seryl residues. In order to determine the exact phosphorylation site, a tandem mass spectrometry (MS/MS) spectrum of the phosphopeptide 
a

$\mathbf{a}_{100}$

\%

b $_{100}$

]

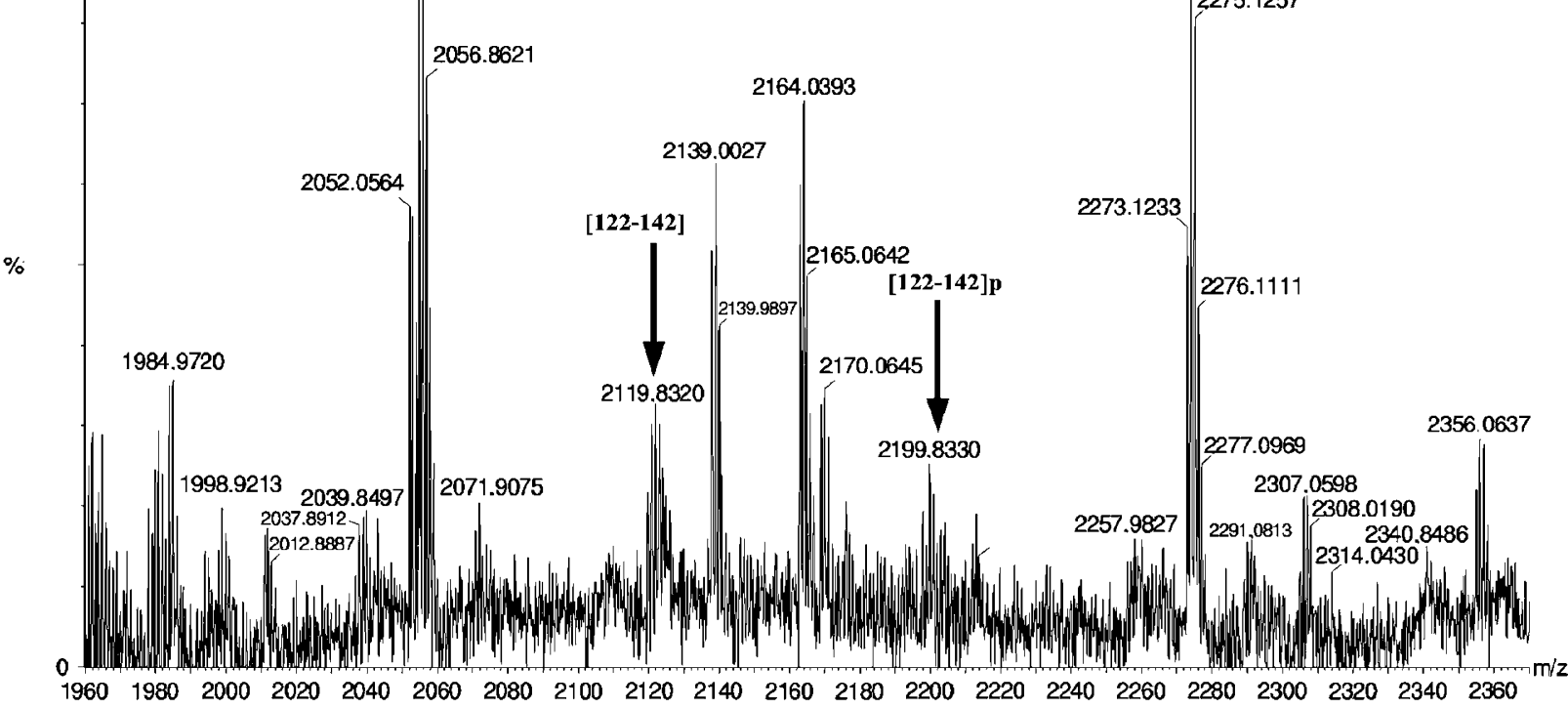

2056.8621
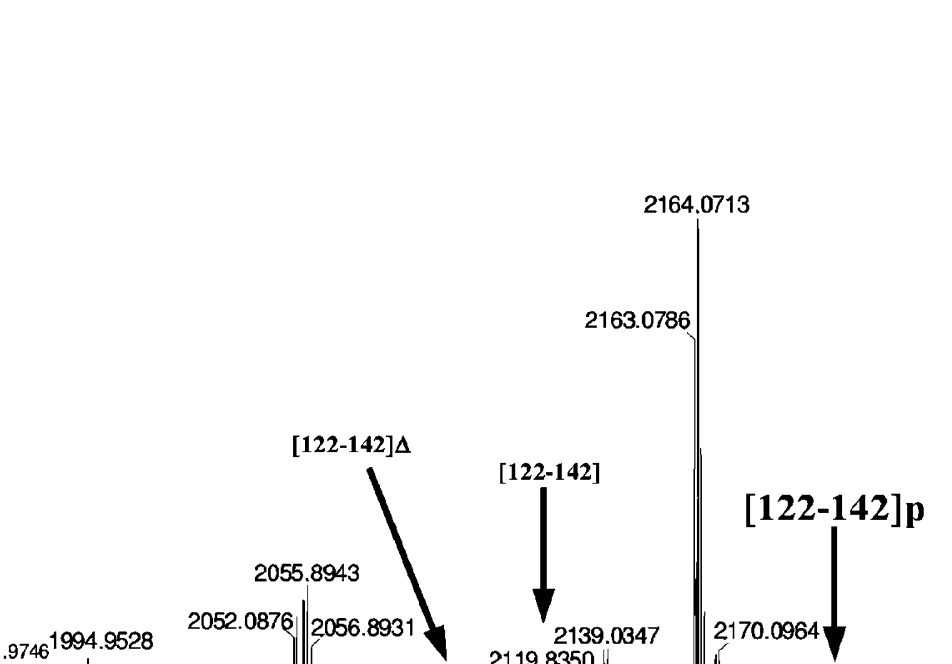

2274,1733

74.1082

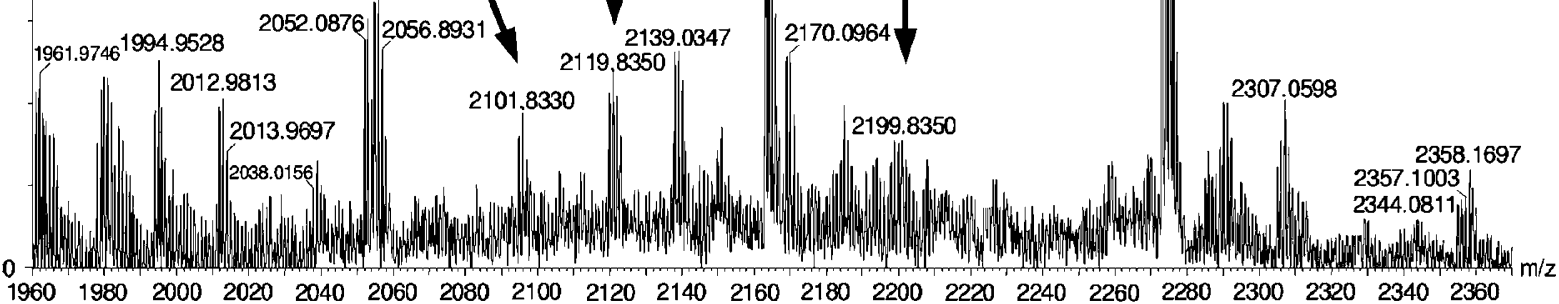

C

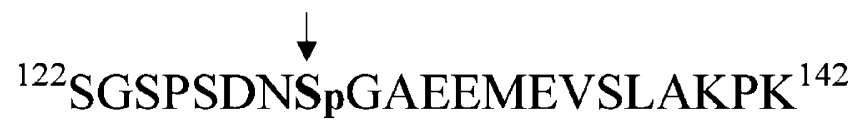

Figure 6 Identification of the CK2 phosphorylation site on Akt. (a) Portion of the MALDI spectrum of Akt1 phosphorylated by CK2 and digested with trypsin as described in Materials and Methods. The peaks corresponding to the tryptic peptide (122-142] (2119.83 Da) and its phosphorylated form [122-142]p (2199.8 Da) are indicated. (b) The same portion of the spectrum of panel (a), after treating the sample with $\mathrm{Ba}(\mathrm{OH})_{2}$. A new peak, indicated as [122-142] $\Delta$ (2101.83 Da), is now evident: it corresponds to the chemically dephosphorylated [122-142] peptide, having a mass shift of $98 \mathrm{Da}$ with respect to the phosphorylated product, due to the $\beta$-elimination of the phosphate group. (c) Sequence of human Akt1 [122-142]. Ser129, target of CK2, is indicated

[122-142] was acquired (not shown). The manual interpretation of the spectrum allowed us to identify Ser129 as the phosphorylated residue.
To confirm the identification of Ser129 as the major target for CK2 in Akt1, we mutated this serine to alanine, and overexpressed both the mutant S129A Akt and the wild-type 


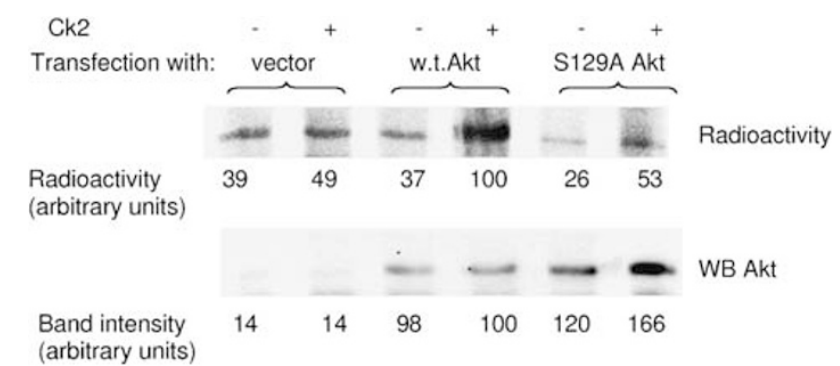

Figure 7 Impaired phosphorylation of the Ser129 Akt mutant by CK2. HEK $293 \mathrm{~T}$ cells were transfected with vector alone, w.t. Akt, or S129A Akt mutant, as indicated. Akt was immunoprecipitated from cell lysates, then incubated in the presence of radioactive phosphorylation mixture, with the addition of $0.1 \mu \mathrm{g}$ of CK2 $\alpha$, where indicated $(+)$. Samples were then loaded on SDS/PAGE followed by blotting, and analyzed by autoradiography (a) or Western blot with anti-Akt antibodies (b). The values reported below each band represent the radioactivity (a), or the intensity of the ECL-developed band (b), and are expressed in arbitrary units

(w.t.) Akt in the highly tarsfectable HEK 293T cell line, reaching a very similar overexpression level in both cases (not shown). We performed experiments where equal amounts of w.t. Akt and S129A Akt were immunoprecipitated and used as substrates for recombinant CK2 in an in vitro phosphorylation assay. As shown in Figure 7, while w.t. Akt, as expected, was readily phosphorylated by $\mathrm{CK} 2$, the phosphorylation of the S129A mutant was severely impaired.

\section{Ser129 is phosphorylated in vivo}

To assess if Ser129 of Akt is phosphorylated in cells, we developed phospho-specific antibodies towards a synthetic phospho-peptide corresponding to amino-acid residues [121140] of human Akt1 sequence, and containing phosphoSer129. The antibodies were first checked in Western blot experiments with the recombinant Akt, phosphorylated or not by $\mathrm{CK} 2$ in vitro. The results, shown in Figure $8 \mathrm{a}$, highlight the specificity of the antibodies towards the phosphorylated form, thus confirming the identification of Ser129 as the target site for CK2 in vitro. When Jurkat cell lysates were analyzed with these antibodies, a weak signal was detected, which became lower in cells treated with TBB (Figure 8b). The response evoked in Western blot by the phospho-Ser129 antibodies was obviously stronger in HEK $293 \mathrm{~T}$ cells transfected with w.t. Akt (Figure 8b); also in this case the signal was significantly decreased by TBB treatment of the cells, while it was absent in cells where the S129A Akt mutant was transfected. In contrast, the signal evoked by an antibody raised again the same region but with nonphosphorylated Ser129 was unchanged.

Parallel experiments were performed by mass spectrometry analysis of the Akt band immunoprecipitated from cells transfected with w.t. Akt: the gel band was treated as described in the Material and Methods section for the in-gel tryptic digestion and analyzed by mass spectrometry. The presence of a phosphorylated form of the peptide [122-142] was confirmed acquiring MALDI MS spectra of the tryptic digest. Again, the acquisition of MS/MS spectra allowed us to confirm the presence of Ser129 as a phosphorylated residue (data not shown).

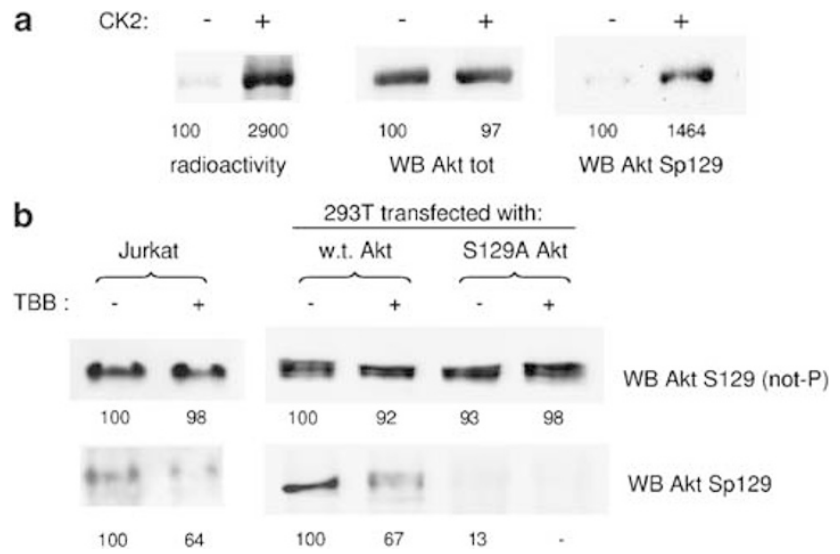

Figure 8 Evidence for Akt Ser129 phosphorylation in vivo. (a) Active Akt $(0.05 \mu \mathrm{g})$ was incubated for $20 \mathrm{~min}$ with radioactive phosphorylation mixture in the absence $(-)$ or in the presence $(+)$ of $0.02 \mu \mathrm{g}$ CK2 $\alpha$. The radioactivity detected by autoradiography, or the signal obtained by Western blot with specific antibodies, are shown, as indicated. (b) Lysates from Jurkat cells or from HEK 293 T cells (transfected with w.t. Akt, or S129A Akt mutant) were analyzed by Western blot with the indicated antibodies. In all, 40 or $10 \mu \mathrm{g}$ of total proteins was loaded, in the case of Jurkat or HEK 293T cells, respectively. Where indicated, TBB $(50 \mu \mathrm{M})$ cell treatment was performed during the last $3 \mathrm{~h}$ before cell lysis. Quantitation of the bands is reported below each band, expressed in arbitrary units, assigning 100 to not treated Jurkat cells, and to not treated 293T cells transfected with w.t. Akt

Since HEK 293T cells do express significant amounts of CK2 (not shown), and considering the decrease of Ser129 phosphorylation observed upon inhibition of CK2 (Figure 8b), we conclude that the w.t. Akt overexpressed from 293 T-cell lysate is phosphorylated at Ser129 in vivo by CK2, although, probably, not stoichiometrically, since it can be further phosphorylated by CK2 in vitro at the same site (see above, Figure 7).

\section{The in vivo activity of the S129A Akt mutant is impaired}

Next we wanted to test if the activity of Akt overexpressed in HEK 293 T cells was impaired by the mutation of Ser129 to Ala. To this purpose, we immunoprecipitated w.t. and S129A Akt and tested their activity towards the Akt peptide substrate. We found that the activity of S129A Akt mutant is markedly reduced, compared to the w.t. enzyme (Figure 9). We also analyzed by Western blot the in vivo phosphorylation state of the Akt substrate GSK3 $\beta$, and of Thr308 and Ser473 of Akt itself, in cells transfected with either w.t. or S129A Akt. We observed a significant reduction of GSK3 phosphorylation in cells transfected with the mutant (Figure 9b). The mutation of Ser129 to Ala also promoted a slight decrease of Ser473 and Thr308 phosphorylation, not sufficient per se to justify the drop in Akt activity.

\section{Discussion}

The data presented disclose a novel functional connection between CK2 and Akt, by showing that, in living cells, downregulation of CK2 catalytic activity or mutation of the 


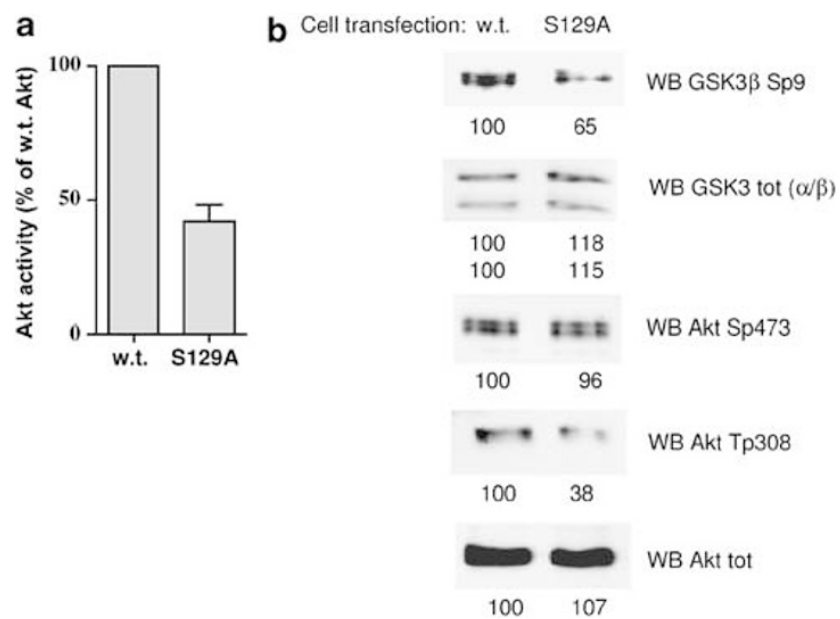

Figure 9 Akt activity in cells transfected with w.t. Akt or Ser129 Akt mutant. HEK 293T cells were transfected with w.t. Akt or S129A Akt mutant, as indicated. (a) Akt was immunoprecipitated from cell lysates, and its activity was measured towards a peptide substrate, by incubation for $30 \mathrm{~min}$ in the presence of a radioactive phosphorylation mixture. The peptide phosphorylation is reported as arbitrary units, assigning an activity of 100 to Akt immunoprecipitated from cells transfected with w.t. Akt. Mean values ( \pm S.D.) from two separate experiments are shown. (b) In all, $5 \mu \mathrm{g}$ (in the case of anti-Akt Sp473) or $20 \mu \mathrm{g}$ (for all the other antibodies) of total lysate proteins was analysed by Western blot (WB) with the indicated antibodies. Band quantitation (arbitrary units, assigning 100 to the signal from cells transfected with w.t. Akt) is reported below each band

CK2-dependent site in Akt correlates with decreased Akt activity, as judged from reduced in vivo phosphorylation of typical Akt targets, and from immunoprecipitate kinase assay.

These data are remarkable inasmuch as they were obtained with PTEN-null cells (either Jurkat or LNCaP) where Akt is already constitutively active and where the possibility is ruled out that CK2 might operate through PTEN, whose in vitro and in vivo phosphorylation by $\mathrm{CK} 2$ has been reported previously. ${ }^{14}$ Also note, in this respect, that the CK2 inhibitors used in this study are ineffective on all isoforms of PI3-kinase ${ }^{20}$ (and unpublished), and they do not affect the activation state of PDK1, supporting the view that CK2 does not impinge on upstream events leading to Akt activation. A direct effect of CK2 on Akt is actually supported by in vitro experiments showing that Akt can be phosphorylated by CK2 with an increase in catalytic activity. Such a stimulation of activity by CK2 could be even more pronounced than it appears from our experiments (Figure 5), considering that in vitro phosphorylation is understoichiometric (see legend of Figure 4).

As expected, CK2 does not phosphorylate Akt at the residues previously reported to trigger Akt activation, notably Thr308 and Ser473, neither of which displays the consensus for CK2. In contrast, CK2 phosphorylates Ser129, a residue located in the linker region between the pleckstrin homology $(\mathrm{PH})$ and the catalytic domains. Ser129 displays the features of a primary target for CK2 (for having an acidic residue at position $\mathrm{n}+3$ ), and its actual phosphorylation has been documented by MS/MS analysis. Ser129 has also been confirmed as the major CK2 target in Akt by its mutation to alanine, which generates a form almost completely unaffected by CK2. Once phosphorylated, Ser129 may trigger the subsequent phosphorylation of Ser126, with a hierarchical mechanism found in several CK2 sites. ${ }^{1}$ Consequently, mutation of Ser129 would also abrogate Ser126 phosphorylation. It can be concluded, therefore, that phosphorylation of Akt at Ser129 (and possibly Ser126) is responsible for hyperactivation of Akt by CK2. Note that Ser129 is conserved in both Akt1 and Akt2. It is not conserved, however, in the related protein kinases SGK, which are not phosphorylated in vitro by CK2.

An in vivo role of CK2-mediated phosphorylation of Akt is strongly supported by data obtained with cells transfected with w.t. or S129A mutant Akt, where the Ser129 phosphorylation is shown to occur in living cells and to correlate with increased activity of Akt.

Pertinent to this may be also the finding that Akt overexpressed in 293 cells contained phosphate constitutively present in the peptide [122-140]; ${ }^{22}$ interestingly, this peptide eluted as a doublet from C18 chromatography, suggesting the presence of multiple phosphorylated forms; indeed, besides the major phospho-aminoacid, identified as Ser124, a weak signal at Ser129 was also reported. While CK2 phosphorylates equally well in vitro either inactive or activated Akt 1 , its favorable effect on activity is evident only with active Akt1, whereas the activity of inactive Akt remains negligible also after phosphorylation by CK2. It may be worth to note, however, that the weak autophosphorylation of inactive Akt1 at Ser473 is significantly enhanced by previous phosphorylation with CK2, supporting the view that this latter event induces anyway conformational changes rendering Akt more prone to activation. Collectively taken, our data suggest that constitutive phosphorylation of Akt by CK2 gives rise to a pool of kinase, which is susceptible to become hyperactive upon phosphorylation of Thr308 by PDK1. Our in vivo experimentation with S129A Akt mutant was performed in a system where Akt is expressed as constitutively active; we therefore cannot discriminate at which level CK2 exerts its role, either before or after canonical activation of Akt triggered by Thr308/Ser473 phosphorylation.

Another point which will deserve additional attention is the weak dephosphorylation of Akt Ser473 and Thr308 observed in cells where CK2 is downregulated. At present, our data do not provide an explanation for this observation; however, it is worth to consider the connection to the Hsp90/cdc37 chaperone system: this complex, known to protect Akt from dephosphorylation, ${ }^{23}$ has been recently demonstrated to be fully competent for its action only if phosphorylated by CK2. ${ }^{24}$ Therefore, this could be a different level of crosstalk between CK2 and Akt. Further studies will be necessary to clarify this issue.

Does stimulation of Akt activity account for the antiapoptotic role of CK2? A definite answer to this question is still premature. On the one hand, there is no doubt that an increment of Akt activity would represent a means to counteract apoptosis, given the central role of this kinase to ensure cell survival. Consistent with this, a Jurkat cell line in which Akt is overexpressed is significantly more resistant to apoptosis induced by CK2 inhibitors than are control Jurkat cells (unpublished), supporting the view that the antiapoptotic effect of CK2 is mediated, at least in part, by Akt.

On the other hand, there is no evidence that stimulation of Akt activity represents the only mechanism by which CK2 promotes cell survival. It should be emphasized that, given its 
extreme pleiotropy, CK2 can impinge on numerous pathways, eventually cooperating with Akt signaling to counteract apoptosis. For example, phosphorylation by $\mathrm{CK} 2$ of $\mathrm{IkB}$ in its PEST region accelerates $\mathrm{lkB}$ degradation by calpain, ${ }^{25-27}$ thus promoting the nuclear translocation of NF-kB and increased expression of antiapoptotic genes. An even more general mechanism by which CK2 can display its antiapoptotic potential would be to render caspase substrates refractory to cleavage: numerous examples are already known of proteins (e.g. Max, Bid, HS1, connexin 45.6, and PTEN) whose phosphorylation by CK2 hinders their cleavage by caspase $^{28}$ (and reviewed in Litchfield ${ }^{9}$ and Pinna ${ }^{29}$ ). But the connection between CK2 and caspases could be even tighter. Besides counteracting the effects of caspases downstream, at substrate level, an upstream device by which CK2 turns off caspase activity has recently been reported, ${ }^{30}$ consisting of the phosphorylation of the caspase-inhibiting protein ARC. Such a phosphorylation is required to target $A R C$ to mitochondria, where it can bind (and inhibit) caspase 8 . Another mechanism by which CK2 may prevent cell death has recently been reported; ${ }^{31}$ the authors show that the proapototic function of CHOP transcription factor is inhibited by $\mathrm{CK} 2-$ mediated phosphorylation.

In the emerging scenario, the newly detected connection with Akt would be a part of a multifaceted strategy by which CK2 coordinates a network of signaling pathways leading to cell survival. Note that some of these, notably caspase inhibition and IkB degradation, could be promoted independently by both CK2 and Akt.

\section{Materials and Methods}

\section{Materials}

\section{Antibodies}

Phospho-GSK3 $\beta$ (S9), phospho-FKHR (S256), which also recognized phospho-AFX (S193), phospho-Akt (T308), and phospho-PDK1 (S241) antibodies were from Cell Signaling Technology, phospho-Akt (S473), total Akt1/2/3, total GSK3 $\beta$ and actin antibodies were from Santa Cruz Biotechnology, CK2 $\alpha$-subunit rabbit antiserum was raised against [376391] region of human protein, corresponding to the specific C-terminal sequence. Phospho-Akt (S129) and its not-phosphorylated counterpart (Akt (S129)) were raised in New Zealand rabbits against two synthetic peptides corresponding to amino acids [121-140] of the Akt protein, phosphorylated in Ser129 or not, respectively; the antisera were purified using an immobilized peptide affinity resin (Sulfo Link Coupling Gel, Pierce, UK) according to the manufacturer's instructions

\section{Kinase substrates}

CK2-specific peptide substrate was synthesized as described elsewhere. ${ }^{32}$ Akt-specific peptide substrate was from Calbiochem, Akt protein substrate piD261 was expressed, purified ${ }^{33}$ and kindly provided by Dr. S Facchin (Padova).

\section{Inhibitors}

TBB was synthesized as in Szyska et al., ${ }^{34}$ IQA, formerly termed CGP029482, ${ }^{20}$ was provided by Novartis; LY294002 was from Cell Signaling Technology, PKA inhibitor peptide was from Sigma.

\section{cDNA constructs}

The expression vector for the murine HA-tagged myrAkt1 (pCMV6-HAmyrAkt1), a costitutively active Akt containing a consensus myristoylation site, was kindly supplied by Dr. Sandra Marmiroli (Modena). S129A Akt mutant was obtained with the 'QuickChange-Site Directed Mutagenesis' Kit (Stratagene) using pCMV6-HA-myrAkt1 as template and two synthetic oligonucleotides primers, 5'-CCCAGTGACAACGCAGGGGCTGAAGA GATGG-3' and 5'-CCATCTCTTCATCCCCTGCGTTGTCACTGGG-3' each complementary to opposite strands of template. The mutation was confirmed by sequencing analysis.

\section{Enzymes}

Active full-length Akt1 (phosphorylated at Thr308 and Ser473) and unactive full-length Akt1 (dephosphorylated) were provided by Dr. $\mathrm{H}$ McLauchlan and Dr. J Hastie (Dundee), recombinant CK2 $\alpha$ and $\alpha_{2} \beta_{2}$ were expressed and purified as in Sarno et al. ${ }^{19}$

\section{Cell culturing and treatment}

The human leukemia Jurkat T-cell line, the human prostate cancer LNCaP cell line, and the human embryonic kidney epithelial HEK 293T cells were maintained in the appropriate medium (RPMI 1640 (Dutch modification, Sigma) for Jurkat and LNCaP, Dulbecco's modified Eagle's medium (Sigma) for HEK 293T, supplemented with 10\% fetal calf serum, $2 \mathrm{mM} \mathrm{L-}$ glutamine, $100 \mathrm{U} / \mathrm{ml}$ penicillin and $100 \mu \mathrm{g} / \mathrm{ml}$ streptomycin, in an atmosphere containing $5 \% \mathrm{CO}_{2}$.

Cell treatments were performed as elsewhere described. ${ }^{11}$

\section{Cell lysis and Western blot analysis}

After treatment, cells were centrifuged, washed, and lysed by the addition of ice-cold buffer consisting of $20 \mathrm{mM}$ Tris- $\mathrm{HCl}, \mathrm{pH} 7.5,150 \mathrm{mM} \mathrm{NaCl}$, $2 \mathrm{mM}$ EDTA, $2 \mathrm{mM}$ EGTA, 0.5\% (v/v) Triton X-100, $2 \mathrm{mM}$ dithiothreitol, protease inhibitor cocktail Complete (Roche), $10 \mathrm{mM} \mathrm{NaF}, 1 \mu \mathrm{M}$ okadaic acid, $1 \mathrm{mM}$ Na vanadate. After $20 \mathrm{~min}$ incubation on ice, the lysates were centrifuged at $10000 \times g$ for $10 \mathrm{~min}$, at $4^{\circ} \mathrm{C}$. The supernatants contained the cell-soluble fraction, whose protein concentration was determined by the Bradford method. Equal amounts of protein were loaded on $11 \%$ SDS/ PAGE, blotted on Immobilon-P membranes (Millipore), and processed in Western blot with the indicated antibody, detected by ECL (enhanced chemiluminescence, Amersham Biosciences). Quantitation of the signal was obtained by chemiluminescence detection on a Kodak Image Station 440CF and analysis with the Kodak 1D Image software.

\section{SiRNA method}

The siRNA sequence targeting specifically human CK2 $\alpha$ containing 21-nt with (2'-deoxy)-tymidine $3^{\prime}$ overhangs and corresponding to nucleotides 803-823 (Accession Number AY112721) was synthesized and annealed by Qiagen-Xeragon Inc. The day before the transfection, cells were diluted with fresh medium without antibiotics and transferred to six-well plates. LNCaP cell transient transfection of SiRNA was carried out according to Elbashir et al., ${ }^{35}$ using Oligofectamine (Invitrogen). In all, $12 \mu$ l culture medium and $3 \mu$ l Oligofectamine per well were preincubated $5 \mathrm{~min}$ at room temperature. During the time of this incubation, $175 \mu \mathrm{l}$ medium were mixed with $10 \mu \mathrm{l}$ siRNA. The two mixtures were combined and incubated for $20 \mathrm{~min}$ at room temperature for complex formation. The entire mixture $(200 \mu l)$ was added to the cells in one well resulting in a final concentration 
of $100 \mathrm{nM}$ for the siRNA. Control cells were treated with $100 \mathrm{nM}$ control nonsilencing siRNA (Qiagen).

\section{Cell transfection}

HEK-293T cells, plated onto 60-mm-diameter dishes at about $80 \%$ confluency, were transiently transfected with $4 \mu \mathrm{g}$ of cDNA by standard calcium-phosphate procedure. The transfection mixture was removed after $16 \mathrm{~h}$, and cells were lysed after $48 \mathrm{~h}$ after transfection.

\section{CK2 activity assay in cell lysates}

In all, $1-2 \mu \mathrm{g}$ of lysate proteins were incubated for $10 \mathrm{~min}$ at $30^{\circ} \mathrm{C}$ with $1 \mathrm{mM}$ CK2-specific peptide RRRADDSDDDDD, in the presence of phosphorylation rection mixture. ${ }^{11}$

\section{In vitro phosphorylation of Akt by CK2}

Purified recombinant Akt $(0.2-0.5 \mu \mathrm{g})$ was incubated with recombinant CK2 ( $\alpha$ or $\alpha_{2} \beta_{2}, 10-20$ units, where 1 unit is the amount of enzyme transferring $1 \mathrm{pmol}$ of $\mathrm{Pi}$ to the peptide substrate per $\mathrm{min}$ ) in the presence of $50 \mathrm{mM}$ Tris- $\mathrm{HCl}, \mathrm{pH} 7.5,12 \mathrm{mM} \mathrm{MgCl}, 10 \mu \mathrm{M} \quad\left[\gamma_{-}{ }^{33} \mathrm{P}\right] \mathrm{ATP}$ ( $\sim 1500 \mathrm{cpm} / \mathrm{pmol}), 0.1 \mathrm{M} \mathrm{NaCl}$, in a total volume of $20 \mu$, for $10 \mathrm{~min}$ at $30^{\circ} \mathrm{C}$.

In the case of overexpressed Akt, Akt was immunoprecipitated from $40 \mu \mathrm{g}$ of lysate proteins with total Akt antibody, following the protocol described elsewhere ${ }^{19}$ the immunoprecipitates were then incubated with CK2, as described for purified Akt.

Samples were run on $11 \%$ SDS/PAGE, and radioactive bands were detected and quantified by autoradiography (Cyclone storage phosphor system, Packard).

For the identification of the amino acids phosphorylated by CK2 in Akt, after phosphorylation samples were hydrolyzed by incubation for $4 \mathrm{~h}$ at $110^{\circ} \mathrm{C}$ in $6 \mathrm{~N} \mathrm{HCl}$, then subjected to high-voltage paper electrophoresis at $\mathrm{pH} 1.9$ for $2.5 \mathrm{~h}$, in the presence of nonradioactive phospho-amino acids, as migration reference. ${ }^{36}$

\section{Akt activity assay}

For Akt immunoprecipitation, 250 or $40 \mu \mathrm{g}$ of total lysate proteins, in the case of Jurkat/LNCaP or HEK 293T cells, respectively, were incubated with total Akt antibody, following the protocol described elsewhere. ${ }^{19}$ Akt immunoprecipitate kinase assay was performed as described in Cross et al., ${ }^{37}$ towards $0.1 \mathrm{mM}$ substrate peptide (RPRAATF), in the presence of $1 \mu \mathrm{M}$ PKA inhibitor peptide. To verify the specificity of the kinase reaction, the activity was also measured in the immunoprecipitates with equal amount of an aspecific antibody, where no Akt was revealed by Western blot analysis.

The activity of purified Akt (specific activity of $1.2 \mathrm{pmol} \mathrm{Pi} / \mathrm{min} / \mu \mathrm{g}$, under basal conditions) was measured towards either the substrate peptide, or the recombinant yeast protein kinase piD261/Bud32, ${ }^{33}$ which contains in its $\mathrm{C}$-terminal tail a site conforming to the consensus sequence of Akt and which is not affected by CK2. Neither the peptide nor piD261 are phosphorylated by CK2 to any appreciable extent. Akt was incubated for $20 \mathrm{~min}$ at $30^{\circ} \mathrm{C}$, with or without $\mathrm{CK} 2$, under the conditions described above, for Akt phosphorylation, with the addition of the substrate $(0.1 \mathrm{mM}$ peptide, or $0.1 \mu \mathrm{g}$ piD261 protein). The substrate phosphorylation degree was detected by means of SDS/PAGE and autoradiography in the case of the protein substrate piD261, or by binding to phospho-cellulose paper and scintillation counting ${ }^{11}$ in the case of the peptide substrate.

\section{Identification of CK2 phosphorylation site in Akt1}

For the determination of the in vitro phosphorylation site, Akt1 was phosphorylated by CK2 and purified by SDS/PAGE. In the case of in vivo phosphorylation, w.t. Akt was overexpressed in 293T cells; before lysis, cells were treated for $2 \mathrm{~h}$ with $1 \mu \mathrm{M}$ okadaic acid, in order to increase the phosphorylation degree; Akt was purified by immunoprecipitation and SDS/PAGE. In both cases, the gel band corresponding to the protein was excised and the protein digestion was performed in gel according to Gharahdaghi. ${ }^{38}$ The digested protein was then analyzed by MALDI mass spectrometry using a MALDI-TOF (Micromass) and $\alpha$-cyano-4-hydroxycinnamic acid as matrix (Sigma). The protein fingerprinting was analyzed with Mascot search engine (Matrix Science, UK) to highlight possible phosphorylated peptides, using Ser/Thr phosphorylation as variable modification. A peptide corresponding to the sequence [122-142] of Akt1 was identified as possibly phosphorylated. The actual phosphorylation was proved chemically dephosphorylating the peptide. $^{39,40}$ The sample was treated with $10 \mu \mathrm{l}$ of $\mathrm{Ba}(\mathrm{OH})_{2}$ for $30 \mathrm{~min}$ at room temperature. The reaction was quenched by adding $2 \mu$ l of formic acid and the sample was desalted using ZipTip (Millipore). The digested protein was then analyzed again by MALDI mass spectrometry and the phosphorylation of the peptide [122-142] was proved by a $98 \mathrm{Da}$ shift of the peak, corresponding to the $\beta$-elimination of phosphate from Ser or Thr residue. The identification of the exact phosphorylated site was made by acquiring an MS/MS spectrum of the phosphopeptide [122-142] using an Ultima Q-TOF (Micromass). The spectrum was manually interpreted according to Hunt et al. ${ }^{41}$ and the position of the phosphorylated site was determined.

\section{Acknowledgements}

We thank Sir Philip Cohen (University of Dundee, Scotland) for critical reading of the manuscript, Joseph Schoepfer (Novartis, Basel, Switzerland) for providing IQA, the protein production team of the Division of Signal Transduction Therapy at the University of Dundee, Scotland (coordinated by Hilary McLauchlan and James Hastie) for providing the active and inactive full-length Akt, Peter James for the availability of the Proteomic Facility at Wallemberg Laboratory II, Lund University (Sweden), Sonia Facchin (University of Padova, Italy) for providing piD261, Sandra Marmiroli (University of Modena e Reggio Emilia, Italy) for providing the cDNA for HA-tagged myrAkt1. This work was supported by grants from the Italian MIUR (PRIN 2003), AIRC, and European Commission (PROKINASERESEARCH 503467).

\section{References}

1. Meggio $F$ and Pinna LA (2003) One-thousand-and-one substrates of protein kinase CK2? FASEB J. 17: 349-368

2. Guerra B and Issinger O-G (1999) Protein kinase CK2 and its role in cellular proliferation, development and pathology. Electrophoresis 20: 20391-20408

3. Tawfic S, Yu S, Wang H, Faust R, Davis A and Ahmed K (2001) Protein kinase CK2 signal in neoplasia. Histol. Histopathol. 16: 573-582

4. Seldin DC and Leder P (1995) Casein kinase II $\alpha$ transgene-induced murine lymphoma: relation to theileriosis in cattle. Science 267: 894-897

5. Kelliher MA, Seldin DC and Leder $P$ (1996) Tal-1 induces $T$ cell acute lymphoblastic leukemia accelerated by casein kinase ll $\alpha$. EMBO J. 15: 5160-5166

6. Landesman-Bollag E, Channavajhala PL, Cardiff RD and Seldin DC (1998) p53 deficiency and misexpression of protein kinase CK2 $\alpha$ collaborate in the development of thymic lymphomas in mice. Oncogene 16: 2965-2974

7. Faust RA, Tawfic S, Davis AT, Bubash LA and Ahmed K (2000) Antisense oligonucleotides against protein kinase CK2- $\alpha$ inhibit growth of squamous cell carcinoma of the head and neck in vitro. Head Neck 22: 341-346 
8. Romieu-Mourez R, Landesman-Bollag E, Seldin DC and Sonenshein GE (2002) Protein kinase CK2 promotes aberrant activation of nuclear factor $-k B$ transformed phenotype, and survival of breast cancer cells. Cancer Res. 62: 6770-6778

9. Litchfield DW (2003) Protein kinase CK2: structure, regulation and role in cellular decisions of life and death. Biochem. J. 369: 1-15

10. Guo C, Yu S, Wang H, Davis AT, Green JE and Ahmed K (2001) A potentia role of nuclear matrix-associated protein kinase CK2 in protection against druginduced apoptosis in cancer cells. J. Biol. Chem. 276: 5992-5999

11. Ruzzene M, Penzo D and Pinna LA (2002) Protein kinase CK2 inhibitor 4,5,6,7tetrabromobenzotriazole (TBB) induces apoptosis and caspase-dependent degradation of haematopoietic lineage cell-specific protein 1 (HS1) in Jurkat cells. Biochem. J. 364: 41-47

12. Vlietstra RJ, van Alewijk DC, Hermans KG, van Steenbrugge GJ and Trapman $J$ (1998) Frequent inactivation of PTEN in prostate cancer cell lines and xenografts. Cancer Res. 58: 2720-2723

13. Shan X, Czar MJ, Bunnell SC, Liu P, Liu Y, Schwartzberg PL and Wange RL (2000) Deficiency of PTEN in Jurkat T cells causes constitutive localization of Itk to the plasma membrane and hyperresponsiveness to CD3 stimulation. Mol. Cell. Biol. 20: 6945-6957

14. Miller SJ, Lou DY, Seldin DC, Lane WS and Neel BG (2002) Direct identification of PTEN phosphorylation sites. FEBS Lett. 528: 145-153

15. Hanada M, Feng J and Hemmings BA (2004) Structure, regulation and function of PKB/AKT - a major therapeutic target. Biochim. Biophys. Acta. 1697: 3-16

16. Scheid MP and Woodgett JR (2003) Unravelling the activation mechanisms of protein kinase B/Akt. FEBS Lett. 546: 108-112

17. Nicholson KM and Anderson NG (2002) The protein kinase B/Akt signalling pathway in human malignancy. Cell. Signal. 14: 381-395

18. Besson A, Robbins SM and Yong VW (1999) PTEN/MMAC1/TEP1 in signal transduction and tumorigenesis. Eur. J. Biochem. 263: 605-611

19. Sarno S, Reddy H, Meggio F, Ruzzene M, Davies SP, Donella-Deana A, Shugar D and Pinna LA (2001) Selectivity of 4,5,6,7-tetrabromobenzotriazole, an ATP site-directed inhibitor of protein kinase CK2 ('casein kinase-2'). FEBS Lett. 496: 44-48

20. Sarno S, de Moliner E, Ruzzene M, Pagano MA, Battistutta R, Bain J, Fabbro D, Schoepfer J, Elliott M, Furet P, Meggio F, Zanotti G and Pinna LA (2003) Biochemical and three-dimensional-structural study of the specific inhibition of protein kinase CK2 by [5-oxo-5,6-dihydroindolo-(1,2-a)quinazolin-7-yl]]acetic acid (IQA). Biochem. J. 374: 639-646

21. Vanhaesebroeck B and Alessi DR (2000) The PI3K-PDK1 connection: more than just a road to PKB. Biochem. J. 346: 561-576

22. Alessi DR, Andjelkovic M, Caudwell B, Cron P, Morrice N, Cohen P and Hemmings BA. (1996) Mechanism of activation of protein kinase $B$ by insulin and IGF-1. EMBO J. 15: 6541-6551

23. Sato S, Fujita N and Tsuruo $T$ (2000) Modulation of Akt kinase activity by binding to Hsp90. Proc. Natt. Acad. Sci. USA 97: 10832-10837

24. Miyata $Y$ and Nishida E (2004) CK2 controls multiple protein kinases by phosphorylating a kinase-targeting molecular chaperone, Cdc37. Mol. Cell. Biol. 24: 4065-4074

25. Lin R, Beauparlant $P$, Makris C, Meloche $S$ and Hiscott J (1996) Phosphorylation of $\mathrm{I}_{\kappa} \mathrm{B} \alpha$ in the $\mathrm{C}$-terminal PEST domain by casein kinase II affects intrinsic protein stability. Mol. Cell. Biol. 16: 1401-1409
26. Shen J, Channavajhala P, Seldin DC and Sonenshein GE (2001) Phosphorylation by the protein kinase CK2 promotes calpain-mediated degradation of $\mid \kappa B \alpha$. J. Immunology 167: 4919-4925

27. Kato Jr T, Delhase M, Hoffmann A and Karin M (2003) CK2 Is a C-Terminal $I_{\kappa} B$ Kinase responsible for NF- $\kappa \mathrm{B}$ activation during the UV response. Mol. Cell. 12: 829-839

28. Torres J, Rodriguez J, Myers MP, Valiente M, Graves JD, Tonks NK and Pulido R (2003) Phosphorylation-regulated cleavage of the tumor suppressor PTEN by caspase-3: implications for the control of protein stability and PTEN-protein interactions. J. Biol. Chem. 278: 30652-30660

29. Pinna LA (2002) Protein kinase CK2: a challenge to canons. J. Cell Sci. 115: 3873-3878

30. Li PF, Li J, Muller EC, Otto A, Dietz R and von Harsdorf R (2002) Phosphorylation by protein kinase CK2: a signaling switch for the caspaseinhibiting protein ARC. Mol. Cell. 10: 247-258

31. Ubeda M and Habener JF (2003) CHOP transcription factor phosphorylation by casein kinase 2 inhibits transcriptional activation. J. Biol. Chem. 278: 4051440520

32. Marin O, Meggio F and Pinna LA (1994) Design and synthesis of two new peptide substrates for the specific and sensitive monitoring of casein-kinase-1 and -2. Biochem. Biophys. Res. Commun. 198: 898-905

33. Facchin S, Lopreiato R, Stocchetto S, Arrigoni G, Cesaro L, Marin O, Carignani $G$ and Pinna LA (2002) Structure-function analysis of yeast piD261/Bud32, an atypical protein kinase essential for normal cell life. Biochem. J. 364: 457-463

34. Szyska R, Grankowski N, Felczak K and Shugar D (1995) Halogenated benzimidazoles and benzotriazoles as selective inhibitors of protein kinases $\mathrm{CK}$ | and CK II from Saccharomyces cerevisiae and other sources. Biochem. Biophys. Res. Commun. 208: 418-424

35. Elbashir SM, Harborth J, Weber K and Tuschl T (2002) Analysis of gene function in somatic mammalian cells using small interfering RNAs. Methods 26 : $199-213$

36. Perich JW, Meggio F, Reynolds EC, Marin O and Pinna LA (1992) Role of phosphorylated aminoacyl residues in generating atypical consensus sequences which are recognized by casein kinase-2 but not by casein kinase-1. Biochemistry 31: 5893-5897

37. Cross DA, Alessi DR, Cohen P, Andjelkovich M and Hemmings BA (1995) Inhibition of glycogen synthase kinase-3 by insulin mediated by protein kinase B. Nature 378: 785-789

38. Gharahdaghi F, Weinberg CR, Meagher DA, Imai BS and Mische SM (1999) Mass spectrometric identification of proteins from silver-stained polyacrylamide gel: a method for the removal of silver ions to enhance sensitivity. Electrophoresis 20: 601-605

39. Meyer HE, Swiderek K, Hoffmann-Posorske E, Korte H and Heilmeyer Jr LM (1987) Quantitative determination of phosphoserine by high-performance liquid chromatography as the phenylthiocarbamyl-S-ethylcysteine. Application to picomolar amounts of peptides and proteins. J. Chromatogr. 397: 113-121

40. Resing KA, Johnson RS and Walsh KA (1995) Mass spectrometric analysis of 21 phosphorylation sites in the internal repeat of rat profilaggrin, precursor of an intermediate filament associated protein. Biochemistry 34: 9477-9487

41. Hunt DF, Yates III JR, Shabanowitz J, Winston S and Hauer CR (1986) Protein sequencing by tandem mass spectrometry. Proc. Natl. Acad. Sci. USA 83: 6233-6237 